\title{
On the hyperbolic Klingenberg plane classes constructed by deleting subplanes
}

\author{
Basri Çelik \\ Dedicated to Professor Hari M Srivastava
}

*Correspondence:

basri@uludag.edu.tr

Department of Mathematics,

Faculty of Arts and Science, Uludag

University, Gorukle, Bursa, Turkey

\begin{abstract}
In this study we investigate the structures constructed by deleting a subplane from a projective Klingenberg plane. If the superplane and the subplane are infinite, then it can be easily seen that the remaining structure satisfies the conditions of a hyperbolic Klingenberg plane. In this study we show that the remaining structure is the hyperbolic Klingenberg plane if the inequality $r \geq m^{2}+m+1+\sqrt{m^{2}+m+2}$ holds when the superplane and the subplane are finite and $t, r$ and $t, m$ are their parameters, respectively.

MSC: 51D20; 05B25
\end{abstract}

Keywords: hyperbolic planes; projective planes; projective Klingenberg planes; finite rings; local rings

\section{Introduction}

There are three kinds of important planes in plane geometry: affine planes, projective planes and hyperbolic planes. In affine planes, only one parallel line can be drawn to a line from a point not on this given line (which is Playfair's version of Euclid's famous fifth postulate, [1]). In projective planes, all lines intersect, that is, we cannot mention parallel lines. In hyperbolic planes, exactly $k$ parallel lines $(k \geq 2)$ can be drawn to a given line from a point not on this given line. In literature, there is a lot of work on these planes.

Geometrical structures, which are more general than affine and projective planes, are obtained by taking a class of points instead of a point, a class of lines instead of a line, and by reorganizing the incidence relation [2]. These generalizations can be found in [3] for affine planes, in [4] for projective planes, and in [5] for hyperbolic planes.

In this paper incidence structures are defined as in [6] and blocks are called lines. For any point $P,(P)$ denotes the set of lines incident with the point $P,[P]$ the cardinality of $(P)$, and $[P, Q]$ the number of lines joining $P$ and $Q .(l),[l]$, and $[l, d]$ are defined dually.

A projective Klingenberg plane (PK-plane) is an incidence structure $\mathcal{K}=(\mathcal{P}, \mathcal{L}, \mathcal{I})$ together with an equivalence relation $\sim$ on $\mathcal{P}$ and $\mathcal{L}$ (called neighbor relation, and the equivalence (neighbor) class of $P$ (resp. $l$ ) is denoted by $\langle P\rangle$ (resp. $\langle l\rangle))$ such that:

(PK1) $P \nsim Q \Longrightarrow[P, Q]=1, \forall P, Q \in \mathcal{P}$.

(PK2) $l \nsim d \Longrightarrow[l, d]=1, \forall l, d \in \mathcal{L}$.

( 2013 Celik; licensee Springer. This is an Open Access article distributed under the terms of the Creative Commons Attribution License (http://creativecommons.org/licenses/by/2.0), which permits unrestricted use, distribution, and reproduction in any medium, provided the original work is properly cited. 
(PK3) There exists a projective plane $\mathcal{K}^{*}$ (the canonical image of $\mathcal{K}$ ) and an incidence structure epimorphism $\varphi: \mathcal{K} \rightarrow \mathcal{K}^{*}$ such that

$$
\begin{aligned}
& P \sim Q \quad \Longleftrightarrow \varphi(P)=\varphi(Q), \quad \forall P, Q \in \mathcal{P}, \\
& l \sim d \Longleftrightarrow \varphi(l)=\varphi(d), \quad \forall l, d \in \mathcal{L} .
\end{aligned}
$$

Axiom (PK3) is equivalent to the following.

(PK3)' Putting $\langle P\rangle \mathcal{I}\langle l\rangle$ iff there are $Q, d$ with $Q \sim P, d \sim l$ and $Q \mathcal{I} d$, the equivalence classes with respect to this incidence an ordinary projective plane $\mathcal{K}^{*}$.

In the above definition, $\nsim$ means 'non-neighboring', and PK-planes are denoted by $\mathcal{K}=$ $(\mathcal{P}, \mathcal{L}, \mathcal{I}, \sim)$.

A point $P$ is said to be near a line $l$, and this is denoted by $P \sim l$, whenever $P \sim Q$ for some $Q \mathcal{I} l$. Detailed information about PK-planes can be found in $[4,7]$.

One can easily prove the following lemma.

Lemma 1 Let $\mathcal{K}=(\mathcal{P}, \mathcal{L}, \mathcal{I}, \sim)$ be a PK-plane. Then

(i) $P \sim l \Longleftrightarrow \exists h \in \mathcal{L}$, such that $h \sim l$, and $P \mathcal{I} h$,

(ii) $h \sim d \Longleftrightarrow \exists H_{i} \mathcal{I} h, \exists D_{i} \mathcal{I} d \ni H_{i} \sim D_{i}, H_{1} \nsim H_{2}, D_{1} \nsim D_{2}, h, d \in \mathcal{L}, H_{i}, D_{i} \in \mathcal{P}, i=1,2$,

(iii) ' $P_{1} \in \mathcal{P}, l_{1}, l_{2} \in \mathcal{L}, P_{1} \mathcal{I} l_{1}, l_{1} \sim l_{2}$ ' $\Rightarrow \exists P_{2} \ni P_{2} \mathcal{I} l_{2}, P_{1} \sim P_{2}$.

When $|\mathcal{P} \cup \mathcal{L}|$ is finite, the geometric structure is called finite. Now, we state a theorem for finite regular PK-planes, which can be found in [8]. The original proof of this theorem for Hjelmslev planes is due to Kleinfeld [9]. Drake and Lenz [10] observed that this proof remains valid for PK-planes.

Theorem 2 Let $\mathcal{K}=(\mathcal{P}, \mathcal{L}, \mathcal{I}, \sim)$ be a PK-plane. Then there are natural numbers $t$ and $r$ which are called the parameters of $\mathcal{K}$, with:

(i) $|\langle P\rangle|=|\langle l\rangle|=t^{2}, \forall P \in \mathcal{P}, l \in \mathcal{L}$.

(ii) $|(P) \cap\langle l\rangle|=|(l) \cap\langle P\rangle|=t, \forall P \mathcal{I} l$.

(iii) Let $r$ be the order of a projective plane $\mathcal{K}^{*}$. If $t \neq 1$, we have $r \leq t$ (then $\mathcal{K}$ is called proper and we have $t=1$ iff $\mathcal{K}$ is an ordinary projective plane).

(iv) $[P]=[l]=t(r+1), \forall P \in \mathcal{P}, \forall l \in \mathcal{L}$.

(v) $|\mathcal{P}|=|\mathcal{L}|=t^{2}\left(r^{2}+r+1\right)$.

A projective or affine Klingenberg plane (PK-, AK-plane) is defined as a generalization of ordinary affine and ordinary projective plane (see [3]). Like these, in [5] the definition for a hyperbolic-Klingenberg plane (HK-plane) is given as a generalization of an ordinary hyperbolic plane. We recall these definitions from the literature.

It is well known that there is an alternative system of axioms for a hyperbolic plane. For instance, Graves introduced the following definition.

A hyperbolic plane is a geometric structure such that:

(A1) There are at least two points on each line.

(A2) Two distinct points lie on one and only one line.

(A3) There exist at least four points, no three of which are collinear.

(A4) Through each point $X$ not on a line $l$, at least two lines pass not meeting (parallel to) $l$. 
(A5) If a subset $\mathrm{S}$ of the points contains all points on the lines through pairs of distinct points of $S$, then the subset $S$ contains all points of the geometric structure (see [11-14]).

A hyperbolic Klingenberg plane (HK-plane) $\mathcal{H}$ is a system $(\mathcal{P}, \mathcal{L}, \mathcal{I}, \|, \sim)$, where $(\mathcal{P}, \mathcal{L}, \mathcal{I})$ is an incidence structure and $\sim$ is an equivalence relation on $\mathcal{P} \cup \mathcal{L}$ (called neighboring) such that no element of $\mathcal{P}$ (point) is neighbor to any element of $\mathcal{L}$ (line), $\|$ is an equivalence relation on $\mathcal{L}$ (called parallelism), and $\mathcal{H}$ satisfies the following axioms for all $P, Q \in \mathcal{P}$, $g, h \in \mathcal{L}:$

(HK1) $P \nsim Q \Longrightarrow[P, Q]=1, \forall P, Q \in \mathcal{P}$.

(HK2) $l \in \mathcal{L} \Longrightarrow \exists P \mathcal{I} l, Q \mathcal{I} l, P \nsim Q$.

(HK3) There exist at least four pairwise non-neighbor points, no three of which are collinear.

(HK4) For each point-line pair $(P, l), P \nsim l$, there are at least two non-neighboring lines through $P$ parallel to $l$.

(HK5) There exist a hyperbolic plane $\mathcal{H}^{*}=\left(\mathcal{P}^{*}, \mathcal{L}^{*}, \mathcal{I}^{*}\right)$ and an incidence structure epimor$\operatorname{phism} \varphi: \mathcal{H} \rightarrow \mathcal{H}^{*}$ such that

$$
\begin{aligned}
& P \sim Q \quad \Longleftrightarrow \varphi(P)=\varphi(Q), \quad \forall P, Q \in \mathcal{P}, \\
& l \sim d \quad \Longleftrightarrow \varphi(l)=\varphi(d), \quad \forall l, d \in \mathcal{L}
\end{aligned}
$$

and if $[g, h]=0$ then $\varphi(g) \| \varphi(h)$ (see [5]).

Various models for hyperbolic planes such as Poincare's models (see [12]), Sandler's models (see [14]) and the extension of Sandler's models (see [13]) have been developed. It is well known that if a line is deleted from a projective plane, then the remaining substructure forms an affine plane. Graves [11], Kaya-Özcan [13] and Sandler [14] gave examples of hyperbolic planes obtained by deletion from projective planes. Sandler showed that if three non-concurrent lines are deleted from a projective plane, then the remaining structure forms a hyperbolic plane [14]. Kaya-Özcan [13] extended Sandler's construction and showed that if $m$ lines, no three of which are concurrent, are deleted from a projective plane, then the remaining structure forms a hyperbolic plane. If $\mathcal{K}$ is a PK-plane and $l$ is a line, by deleting all lines neighbor to $l$ and all points near to $l$, the remaining structure forms an affine Klingenberg plane. In [5] the method of [13] was adopted for obtaining an HK-plane from a PK-plane.

\section{Deleting subplane from finite PK-plane}

Let $\Pi=(\mathcal{P}, \mathcal{L}, \mathcal{I})$ be a finite projective plane of order $n$, then it is well known that $\Pi$ has $n^{2}+n+1$ points and $n^{2}+n+1$ lines. Also, there are $n+1$ points on each line and $n+1$ lines pass through each point. If $\Pi$ is any finite projective plane, then the possible order of subplanes of $\Pi$ is restricted by the following theorem (see [15]).

Theorem 3 Let $\Pi$ be a finite projective plane of order $n$ with a proper subplane $\Pi^{\prime}$ of order $m$. Then either $n=m^{2}$ or $n \geq m^{2}+m$. If $n=m^{2}$, a subplane is called a Baer subplane.

The following lemma is proven by simple calculations (see [15]). 
Lemma 4 If a Baer subplane with order $m$ is deleted from the projective plane of finite order $n$, then the remaining structure is empty.

The following theorem is given from [16].

Theorem 5 Let $\Pi=(\mathcal{P}, \mathcal{L}, \mathcal{I})$ be a finite projective plane of order $n$ with a non-Baer subplane $\Pi^{\prime}=\left(\mathcal{P}^{\prime}, \mathcal{L}^{\prime}, \mathcal{I}^{\prime}\right)$ of order $m$. Then the substructure $\Pi_{0}=\left(\mathcal{P}_{0}, \mathcal{L}_{0}, \mathcal{I}_{0}\right), \mathcal{P}_{0}=\mathcal{P} \backslash\{X \in$ $\left.\mathcal{P} \mid X \in l, l \in \mathcal{L}^{\prime}\right\}, \mathcal{L}_{0}=\mathcal{L} \backslash \mathcal{L}^{\prime}, \mathcal{I}_{0}=\mathcal{I} \cap\left(\mathcal{P}_{0} \times \mathcal{L}_{0}\right)$ is a hyperbolic plane if $n \geq m^{2}+m+1+$ $\sqrt{m^{2}+m+2}$.

Now we can adopt the last theorem to obtain a hyperbolic Klingenberg plane from a projective Klingenberg plane. However, we must give the following lemma before the theorem. Since the incidence relation and neighboring are similar, we use the same notation for the incidence and neighbor relations of a subplane and a superplane.

Lemma 6 Let $\mathcal{K}=(\mathcal{P}, \mathcal{L}, \mathcal{I}, \sim)$ be a finite projective Klingenberg plane with parameters $t$ and $r$, and let $\mathcal{K}^{\prime}=\left(\mathcal{P}^{\prime}, \mathcal{L}^{\prime}, \mathcal{I}, \sim\right)$ be a subplane of $\mathcal{K}$ with parameters $t, m$ such that $r \geq$ $m^{2}+m$. Then we consider the substructure $\mathcal{K}_{0}=\left(\mathcal{P}_{0}, \mathcal{L}_{0}, \mathcal{I}, \sim\right)$, where $\mathcal{P}_{0}=\mathcal{P} \backslash\{X \in \mathcal{P} \mid \exists l \in$ $\left.\mathcal{L}, l \cap \mathcal{P}^{\prime}=l^{\prime} \in \mathcal{L}^{\prime}, X \in l\right\}$, then the following properties hold:

(i) For any $l \in \mathcal{L},\langle l\rangle$ has $t^{2}(r+1)$ points.

(ii) If $P \nsim Q$ and $P, Q \in \mathcal{P}_{0}$, then $[P, Q]=1$.

(iii) $\left|\mathcal{L}_{0}\right|=t^{2}\left(r^{2}+r-m^{2}-m\right)$.

(iv) $\left|\mathcal{P}_{0}\right|=t^{2}(r-m)\left(r-m^{2}\right)$.

(v) If $l_{0} \in \mathcal{L}_{0}$, then $\left[l_{0}\right]=t\left(r-m^{2}-m\right)$ or $\left(l_{0}\right)=t\left(r-m^{2}\right)$.

Proof (i) For any $d \in\langle l\rangle, \varphi(d)=d^{*}$ is a line of $\mathcal{K}^{*}$ which is the projective plane of order $r$ where $\varphi: \mathcal{K} \rightarrow \mathcal{K}^{*}$ is the incidence structure epimorphism given in (PK3). Since $d^{*}$ has $r+1$ points and every point of $\mathcal{K}^{*}$ is the image of $t^{2}$ neighbor points, $\langle l\rangle$ has $t^{2}(r+1)$ points.

(ii) Since $\mathcal{P}_{0} \subset \mathcal{P}$, it is obvious from (PK1) for $\mathcal{K}$.

(iii) Since $\mathcal{K}$ and $\mathcal{K}^{\prime}$ has $r^{2}+r+1$ and $m^{2}+m+1$ pairwise non-neighbor lines respectively, $\mathcal{K}_{0}$ has

$$
r^{2}+r+1-\left(m^{2}+m+1\right)=r^{2}+r-m^{2}-m
$$

pairwise non-neighbor lines. Therefore $\mathcal{K}_{0}$ has $t^{2}\left(r^{2}+r-m^{2}-m\right)$ lines.

(iv) Let $l^{\prime} \in \mathcal{K}^{\prime}$ and $l \in \mathcal{K}$ be such that $l^{\prime}=l \cap \mathcal{P}^{\prime}$, then $\left\langle l^{\prime}\right\rangle$ has $t^{2}(m+1)$ points and $\langle l\rangle$ has $t^{2}(r+1)$ points. For this reason, $\langle l\rangle$ has $t^{2}(r-m)$ points which do not belong to $\left\langle l^{\prime}\right\rangle$. Since $\mathcal{K}^{\prime}$ has $m^{2}+m+1$ pairwise distinct neighbor classes for lines, there are $t^{2}(r-m)\left(m^{2}+m+1\right)$ points belonging to deleted lines not in $\mathcal{P}^{\prime}$. In addition to these points, all the points of $\mathcal{K}^{\prime}$ are deleted. Therefore the total number of deleted points is

$$
t^{2}(r-m)\left(m^{2}+m+1\right)+t^{2}\left(m^{2}+m+1\right)=t^{2}\left(m^{2}+m+1\right)(1+r-m) .
$$

Consequently, $\mathcal{K}_{0}$ has

$$
t^{2}\left(r^{2}+r+1\right)-t^{2}\left(m^{2}+m+1\right)(1+r-m)=t^{2}(r-m)\left(r-m^{2}\right)
$$

points. 
(v) Let $l_{0}=l \cap \mathcal{P}_{0} \in \mathcal{L}_{0}$ and $l \in \mathcal{L}$. There exist two cases: $l \cap \mathcal{P}^{\prime}=\phi$ or not.

(a) Let $l \cap \mathcal{P}^{\prime}=\phi$. Then $[l]=t(r+1)$ and all pairwise non-neighbor lines of $\mathcal{K}^{\prime}$ intersect $l$ on pairwise non-neighbor points. Since $m^{2}+m+1$, the pairwise distinct lines of $\mathcal{K}^{\prime}$ intersect $l$ on $t\left(m^{2}+m+1\right)$ points which are deleted from $l$. Therefore $\left[l_{0}\right]=t(r+1)-$ $t\left(m^{2}+m+1\right)=t\left(r-m^{2}-m\right)$.

(b) Let $l \cap \mathcal{P}^{\prime} \neq \phi$. Then there exists a point $N \in \mathcal{P}^{\prime}$ such that $l \cap \mathcal{P}^{\prime}=l \cap\langle N\rangle$. Therefore $l \cap \mathcal{P}^{\prime}$ has $t$ neighbor points. The number of pairwise non-neighbor lines of $\mathcal{K}^{\prime}$ through the points neighbor to $N$ is $m+1$. Since $\mathcal{K}^{\prime}$ has $m^{2}+m+1$ pairwise distinct lines and $m+1$ of these lines pass through the neighbor points to $N$, there are $m^{2}$ pairwise nonneighbor lines in $\mathcal{K}^{\prime}$ which intersect $l$ on pairwise non-neighbor points not belonging to $\langle N\rangle$. Therefore $m^{2}+1$ point neighbor classes are deleted from $l$. Since every neighbor class of points has $t$ points on $l$, we have $\left[l_{0}\right]=t\left(r+1-\left(m^{2}+1\right)\right)=t\left(r-m^{2}\right)$.

Under the same assumptions of the following theorem, in [16] it is shown that $\mathcal{K}_{0}^{*}$ is the hyperbolic plane, and therefore we obtain the following theorem considering the previous lemma.

Theorem 7 The structure $\mathcal{K}_{0}$ given in the previous lemma is a hyperbolic Klingenberg plane if $r \geq m^{2}+m+1+\sqrt{m^{2}+m+2}$.

\section{Some outstanding problems}

In this paper, it is shown that the structure obtained by deletion of a subplane with parameters $t, m$ from the finite Klingenberg plane with parameters $t, r$ is a hyperbolic Klingenberg plane when the parameters of the subplane are suitably small relative to the parameters of the superplane. But now we give some outstanding problems.

- When is the structure $\mathcal{K}_{0}$ a HK-plane where the parameters of the superplane are $t, r$ and the parameters of the subplane are $t^{\prime}, m$ and $t \neq t^{\prime}$ ?

- When is a HK-plane with appropriate parameter restriction a subplane deleted PK-plane?

Competing interests

The author declares that he has no competing interests.

\section{Acknowledgements}

This work was supported by the Commission of Scientific Research Projects of Uludag University, Project number UAP(F)-2012/23.

Received: 11 January 2013 Accepted: 12 July 2013 Published: 29 July 2013

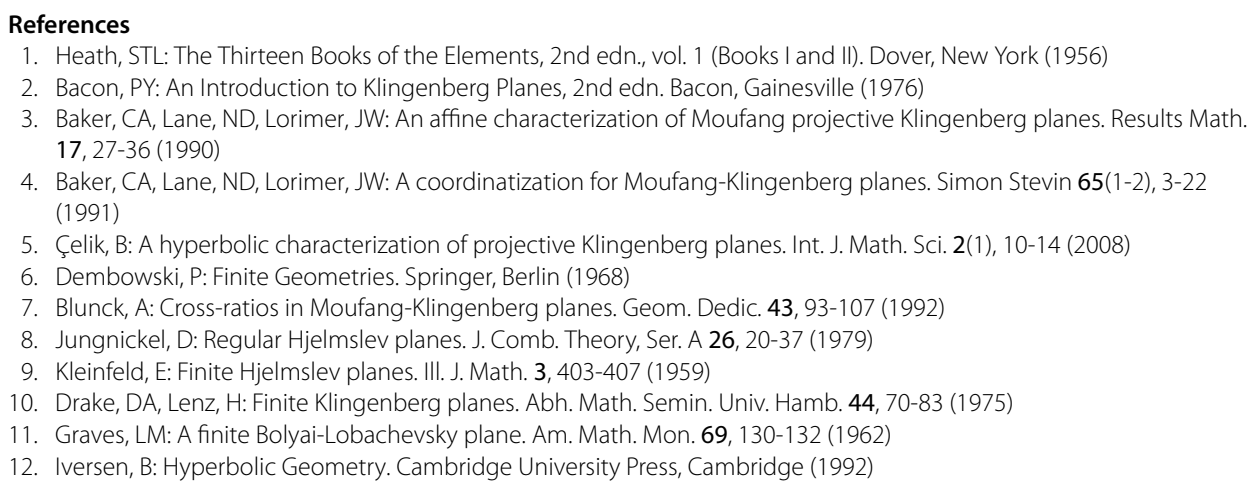


13. Kaya, R, Özcan, E: On the construction of Bolyai-Lobachevsky planes from projective planes. Rend. Semin. Mat. Brescia 7, 427-434 (1982)

14. Sandler, R: Finite homogeneous Bolyai-Lobachevsky planes. Am. Math. Mon. 70, 853-854 (1963)

15. Hughes, DR, Piper, FC: Projective Planes. Springer, Berlin (1973)

16. Çelik, B: On some hyperbolic planes from finite projective planes. Int. J. Math. Math. Sci. 25(12), 757-762 (2001)

doi:10.1186/1029-242X-2013-357

Cite this article as: Çelik: On the hyperbolic Klingenberg plane classes constructed by deleting subplanes. Journal of Inequalities and Applications 2013 2013:357.

Submit your manuscript to a SpringerOpen ${ }^{\circ}$ journal and benefit from:

- Convenient online submission

Rigorous peer review

- Immediate publication on acceptance

- Open access: articles freely available online

- High visibility within the field

- Retaining the copyright to your article

Submit your next manuscript at $>$ springeropen.com 